\title{
Le langage des ruines réactualisé par la Grande Guerre
}

Résumé: Qu'advient-il du « langage des ruines », expression portée par le romantisme, dans le contexte de la Première Guerre mondiale? L'analyse d'un corpus de textes d'auteurs contemporains du conflit, de langue anglaise et française, permet de mesurer l'incidence du changement d'objet (du vestige au décombre) sur le discours et sur l'imaginaire collectif. Le spectacle des destructions architecturales sans précédent perpétrées sur le front occidental agit sur le contenu sémantique et sur les implications du mot « ruine » tout en recomposant le jeu des références. Les discours de protestation font apparaître une évolution dans la relation au patrimoine monumental qui se décline en trois dimensions conflictuelles (nationale, locale, mondiale). Enfin, il importe de savoir si le plaisir esthétique est compatible avec cette épreuve de la perte et du deuil et s'il est possible de parler d'une poétique de la ruine de guerre dans les œuvres des écrivains-combattants. Ainsi, les données nouvelles de la guerre industrielle et de la mort de masse contribuent à la réécriture $\mathrm{du}$ « langage des ruines ».

Mots clés: Ruines de guerre, Première Guerre mondiale, destructions architecturales, esthétique des ruines, monuments historiques, patrimoine de l'humanité

C’est par le détour de la métaphore que la présente communication s’inscrit dans la thématique générale du Congrès, centrée sur « le langage » : " On a parlé quelquefois du langage des ruines », peut-on lire dans la correspondance de deux auteurs français, qui reprennent en 1830 une expression portée par le romantisme. Ils recourent à ce langage poétique pour exprimer leur émotion à la découverte des vestiges d'une lointaine cité orientale ${ }^{1}$.

Peut-on, sans risque d'anachronisme voire d'inconvenance, se référer à la tradition esthétique et littéraire pour évoquer des ruines, non pas poétisées par l'exotisme et le passage du temps mais celles, brutales et sans précédent, produites par un conflit moderne et particulièrement meurtrier ? Nous verrons, à la lecture des textes d'auteurs contemporains, que la réutilisation de la formule

1 « On a parlé quelquefois du langage des ruines ; ce langage que les âmes poétiques peuvent seules comprendre, révèle des secrets qui ne sont point du domaine de l'histoire, et qui ont souvent bien plus d'intérêt et de charme que l'histoire elle-même. » (Michaud et Poujoulat 1841, 42 ; Lettre LXXV : Ville de Guzel-Hissar. Ruines de Tralles).

2 Open Access. (C) 2021 Joëlle Prungnaud, published by De Gruyter. (c) BY-NC-ND This work is licensed under the Creative Commons Attribution-NonCommercial-NoDerivatives 4.0 International License. 
dans le contexte de la Première guerre mondiale ne manque pourtant pas de pertinence. Cette perspective peut, en effet, nous éclairer sur les évolutions culturelles en cours, notamment sur la relation au patrimoine et sur la position des écrivains dans ce domaine.

Il sera question de langage verbal (et non iconographique), c'est-à-dire de ce que disent les ruines - du moins ce qu'on leur fait dire -, et de ce qu'on dit sur les ruines : un discours de et un discours sur, à replacer dans la guerre des mots, quand la violence belliciste se déplace sur le terrain de l'art et de la culture et quand elle est relayée par le monde des lettres. Les exemples seront puisés dans un corpus de langue anglaise et française, émanant des protagonistes d'un même camp face à l'ennemi commun.

Les destructions de la Seconde guerre mondiale ont eu tendance à occulter et à minimiser, par comparaison, le souvenir de celles de 14-18. Il est vrai que les dégâts causés par l'artillerie lourde n'eurent pas la même ampleur que ceux causés par les bombardements aériens généralisés sur les territoires de tous les belligérants en 39-45. La zone des combats (de la mer du Nord à la frontière suisse, pour s'en tenir au front Ouest) fut, certes, limitée mais soumise à un pilonnage intense et constamment renouvelé. En conséquence, la confrontation avec les spectacles de désolation venus du front fut à la fois soudaine et durable, puisqu'elle se prolongea jusque dans les années d'après-guerre. C'est l'inédit d'une destruction à une telle échelle qui fait événement ainsi que la donnée nouvelle de l'autodestruction une fois le front stabilisé en territoire français et belge. Dès le mois d'août 1914, les ruines de la Belgique envahie témoignent de la violence exercée contre la population civile et prouvent que le conflit s'engage dans le mépris des lois de la guerre censées préserver les biens culturels ${ }^{2}$. Il faut se remettre dans l'état d'esprit des contemporains pour mesurer la stupeur provoquée par le sac de Louvain puis par l'incendie de la cathédrale de Reims dès les premiers mois de combat. $\mathrm{Au}$ traumatisme causé par les pertes humaines, venait s'ajouter le choc de l'atteinte portée aux monuments historiques après un siècle de luttes contre toutes les formes de vandalisme et la création d'institutions chargées de la protection du patrimoine, au niveau européen.

2 L'article 27 de la IV Convention de La Haye, du 18 octobre 1907, stipule : « Dans les sièges et bombardements, toutes les mesures nécessaires doivent être prises pour épargner, autant que possible, les édifices consacrés aux cultes, aux arts, aux sciences et à la bienfaisance, les monuments historiques, les hôpitaux et les lieux de rassemblement de malades ou de blessés, à condition qu'ils ne soient pas employés en même temps à un but militaire ", in Deuxième conférence internationale de la paix, 1907 (1908, 209). 
Nous verrons, dans un premier temps, que cet effet de surprise et de sidération agit directement sur le choix des mots, sur la quête de références, dans les commentaires et les descriptions. Un effort est manifestement fait pour intégrer les données nouvelles au discours convenu sur les ruines et pour prendre en compte le changement de statut qui s'opère, du vestige archéologique à la ruine de guerre.

De 1914 à 1918, et au-delà de l'Armistice, les protestations contre les destructions suscitent une inflation de discours utilisés par la propagande des deux camps ennemis. Le manichéisme de l'affrontement et l'exploitation de l'indignation par les autorités ont réduit cette somme de réactions à un argumentaire constamment répété et mis en avant. Pour trouver un intérêt à cette relecture aujourd'hui, il faut briser cette uniformité de façade et relever au contraire les contradictions qui sous-tendent ces prises de position.

Nous interrogerons enfin la spécificité du discours inspiré ou produit par la ruine de guerre : la perspective esthétique est-elle exclue du fait de ce statut ? Que deviennent les catégories héritées du romantisme : le pittoresque, le sublime ? C’est ici que se pose la question de la créativité littéraire dans le traitement de cette architecture meurtrie : le nouvel imaginaire de la ruine mène-t-il à de nouvelles formes d'expression?

\section{Du vestige archéologique à la ruine de guerre}

Voyons tout d'abord de plus près le mot-clé de cette analyse : le mot "ruines » lui-même, à travers ses occurrences dans les textes de l'époque. Son usage et son contenu sémantique évoluent sous la pression des événements. Pour les contemporains confrontés aux images des zones dévastées du front, le mot ne correspond plus à l'image collective et intériorisée de la ruine. Celle-ci pourrait se définir comme un ensemble formel circonscrit, balisé, archivé par une culture de la conservation qui tend à sacraliser la trace matérielle de l'histoire ${ }^{3}$. Comme si la réalité ne coïncidait plus avec l'imaginaire, il se produit un phénomène de décalage, de dépaysement qui oblige à requalifier l'objet. D'où un effort de l'expression pour creuser l'écart avec les ruines archéologiques ou historiques, ne serait-ce que par l'addition de qualificatifs qui marquent un tournant lié à une prise de conscience : en 1915, l'écrivain d'art Robert de La Sizeranne parle de « ruines

3 C'est la thèse de l'historien d'art viennois Aloïs Riegl, exposée dans Der moderne Denkmalkultus (Riegl 1903), trad. fr. Le Culte moderne des monuments. Son essence et sa genèse (Riegl 1984). 
neuves » (La Sizeranne 1915). Pierre Loti de « ruines toutes fraîches » (Loti 1917, 4). Un poète-soldat publie en 1917 une plaquette intitulée : Jeunes Ruines. Poëmes de guerre (Selthic, 1917). Henri Lavedan ${ }^{4}$, dans la préface de ce recueil, salue la force expressive de cette « trouvaille » (qui n'en est pas une, en fait, car on la trouve déjà dans deux romans d'Émile Zola ${ }^{5}$ et avant lui chez Théophile Gautier ${ }^{6}$ ). L'oxymore souligne le côté tragique du raccourci temporel, rappelle que la destruction ne s'inscrit pas dans le cycle long de l'histoire mais dans la violence brutale du présent. Il est clair, à la lecture de ces pages, que la jeunesse combattante (le poète et l'illustrateur) se projette dans ces «jeunes ruines », auxquelles feront écho les « jeunes morts » de Roland Dorgelès ${ }^{7}$.

La nouveauté de la chose vue tient aussi aux proportions, à la question de l'échelle bien exprimée encore par Robert de La Sizeranne $(1918,617)$ : «Sur les champs de bataille, on voyait çà et là une ruine : maintenant ce sont des paysages de ruines ». La dévastation s'étend à la nature (saccage des arbres, champs troués par les obus), ce qui le conduit à parler de « ruines végétales » (La Sizeranne 1915). Élie Faure résume : « la terre même est une ruine » (Faure 2005, 213214). Tous cherchent des termes de substitution pour marquer la rupture avec le vocabulaire de l'esthétique : Maeterlinck intitule son recueil d'articles de 1916 Les débris de la guerre (Maeterlinck 1916), tandis que d'autres peinent à nommer les restes de Gerbéviller, un des premiers villages français anéantis : « ce ne sont pas là des ruines, ce sont des décombres » (La Sizeranne 1915), « décombres sans nom ", ajoute Maurice Barrès $(1919,2)^{8}$. Le critique anglais Edmund Gosse insiste sur le passage à l'informe : le Beffroi d’Arras a été transformé en une ruine confuse [« confused ruin »], les maisons de la Grand-Place réduites à un monceau de poussière ["shapeless dust-heap »] ; quant aux édifices religieux, il n'en reste que des déchets impossibles à identifier [ u unrecognisable rubbish »] (Gosse 1916, 90-91). On note l'application de Rudyard Kipling à rendre les effets de la désintégration dans le chœur de la cathédrale de Reims : il ne voit que « verre pilé, pierre réduite en poudre, lambeaux de plomb, fragments de fer » [« dust of glass, powdered stone, twists of leading, iron fragments »], c'est-àdire un effacement de toute création formelle (Kipling 2014, 39 ; 1915, 27). Cette

4 Henri Lavedan (1859-1940), chroniqueur, dramaturge et romancier. Membre de l'Académie française depuis 1898.

5 Lourdes (Zola 1995, 346) et Rome (Zola 1999, 405) pour désigner les ruines modernes, en l'occurrence un chantier de construction abandonné.

6 Gautier $(1881,338)$ : « cette jeune ruine faite de main d'homme », à propos de l'Hôtel de Ville de Paris incendié sous la Commune.

7 Dorgelès $(2010,121)$ : le cadavre exhumé d'un soldat est qualifié de « jeune mort ».

8 On retrouve la même expression chez Élie Faure $(2005,213)$ : « débris sans noms ». 
métamorphose est due aux nouveaux « dieux de la guerre » selon Maeterlinck, qui appartiennent au « monde informe et brut de la matière inerte ${ }^{9}$, celui de la chimie, de l'acier, de l'industrie lourde (associé à l'Allemagne). C'est la victoire du fer contre la pierre ${ }^{10}$, de la science des ingénieurs contre l'art, le triomphe de la guerre de matériel sur le vivant et le sensible à l'origine du geste créateur. Le langage des ruines est ainsi porteur des controverses qui ont agité le monde culturel au tournant du siècle.

Qu'est-ce qui est en jeu dans ces efforts de re-nomination ? Sans doute la volonté de marquer le changement, de montrer la rupture : du déjà vu au jamais vu. Mais, dans le même temps, et dans une démarche qui contredit la proclamation de l'inédit, on se met en quête d'une antériorité en se référant à des modèles du passé (récent, antique ou intemporel).

Par exemple, l’Anglaise Vernon Lee compare sa consternation à la vue de la ville de Reims détruite par l'artillerie allemande à celle éprouvée dans son adolescence devant les restes calcinés des Tuileries et de l'Hôtel de Ville incendiés sous la Commune (Lee 1925, 243). Il est significatif qu'elle fasse appel à sa mémoire plutôt qu'à l'histoire pour transmettre le choc éprouvé. On peut s'étonner que le rappel de ces ruines en plein Paris soit plus fréquent que celui des exemples laissés par la guerre de 1870. La Cour des Comptes est, en effet, souvent citée (Péladan 1917, 175, 278), sans doute parce que ses ruines spectaculaires restèrent exposées au regard des Parisiens pendant plus de vingt ans. En revanche, les dégâts commis lors de l'invasion des Prussiens n'ont pas eu le même retentissement dans l'opinion, en dépit du bombardement de la cathédrale de Strasbourg ${ }^{11}$, et alors que les descriptions de Zola annoncent celles de 14-18, notamment quand le romancier évoque ce village proche de Sedan :

[...] ce Bazeilles si riant trois jours plus tôt, avec ses gaies maisons au milieu de ses jardins, à cette heure effondré, anéanti, ne montrant que des pans de muraille noircis par les flammes. (Zola 1984, 386-387)

9 Maeterlinck, « Les Dieux de la guerre » $(1916,156)$. Texte antérieur à la guerre (publié dans De l'Intelligence des fleurs, 1907), que l'auteur a jugé assez pertinent pour compléter le recueil de 1916.

10 Lavedan $(1919,95)$ : « sous des kilogrammes de fer, les barbares ont crevé [...] la merveille de pierre $"$.

11 Théophile Gautier $(1881,5)$ : « [...] une pluie de fer tombe nuit et jour sur le Münster, brisant les clochetons, mutilant les statuettes, perçant les voûtes des nefs [...]. La bibliothèque, unique au monde en son genre, a brûlé ». 
Se manifeste ici l'effet saisissant produit par la juxtaposition d'un avant et d'un après : à la gaieté du temps de paix succède sans transition le sombre spectacle de la désolation. L'ellipse de l'attaque par le feu renforce la brutalité du contraste entre les deux situations. Ce procédé sera souvent utilisé dans les témoignages de la Grande Guerre et plus encore dans l'iconographie (la même prise de vue, hier et aujourd'hui).

À l'opposé de cet ancrage dans l'immédiateté du contemporain, on relève le besoin de se référer à l'aube des temps devenus légendaires pour prendre la mesure de la catastrophe. Ainsi, Paul Valéry cite les ruines des puissants royaumes évoqués dans la Bible : «Élam, Ninive, Babylone ${ }^{12}$ » pour tirer, en 1919, la terrible leçon de la guerre : « Nous autres, civilisations, nous savons maintenant que nous sommes mortelles » (Valéry 1957, 988). De même, les grands cataclysmes naturels sont souvent convoqués, en particulier celui de Pompéi ${ }^{13}$. Si le choix des références peut être un indicateur du traumatisme vécu par les contemporains ou d'une interprétation distanciée du conflit (comme châtiment universel, dans la fatalité de la répétition), il est aussi lourd d'implications politiques.

En témoignent les fluctuations du discours sur l'édifice devenu emblématique des destructions de 14-18 en France : la cathédrale de Reims, régulièrement bombardée pendant toute la durée du conflit. Au lendemain de l'incendie du 19 septembre 1914 provoqué par des obus allemands, un communiqué officiel du gouvernement annonce : « À cette heure, la basilique n'est plus qu'un monceau de ruines ${ }^{14}$ » (Harlaut 2001, 74). Cette fausse nouvelle est ensuite reprise (sans vérifications) et amplifiée par la presse (voir Danchin 2015, 64-65). Il en reste une trace durable dans les recueils de chroniques et de textes d'auteurs publiés après la guerre. C'est le cas de la publication d'Henri Lavedan, qui n'apporte aucun correctif, en 1919, à ses assertions de 1914 : " Cette sublime demeure [...] n'est plus aujourd'hui qu'un puits à ciel ouvert au fond duquel s'entassent broyées les merveilles de l'art » (1919, 95 ; notons au passage la vertigineuse inversion de la verticalité gothique en profondeur abyssale). Le fantasme collectif de l'écroulement persiste dans les esprits, en dépit des faits : « La cathédrale de Reims est fumante, croulante, noircie : ses voûtes tiennent encore ", écrit Émile Mâle en octobre 1914, « mais qu'un hiver passe, et ce sera une grande ruine désolée, où l'on n'entendra d'autre bruit que celui des pierres qui, les unes après les autres,

12 Puissants royaumes réduits en cendre par la colère divine : Élam, Jérémie, 25, 25 ; Babylone, ibid., 50 ; Ninive, Tobie, $1,10$.

13 Rudyard Kipling $(1915,31)$ : « There was a village that had been stamped flat, till it looked older than Pompeii. »

14 Propos du ministre des Affaires étrangères Delcassé. 
se détachent et tombent » (Mâle 1918, 220). Parallèlement à ce discours alarmiste de déploration émerge et s'affirme le registre de l'éloge, au point que la ruine fantasmée s'érige en temple grec : elle sera désormais « pour nous un Parthénon ! », comme le proclame Edmond Rostand, c'est-à-dire « Le Symbole du Beau consacré par l’insulte!» (Rostand 1914, 115). L’idée sera reprise ensuite et répétée comme un leitmotiv jusqu'à devenir dès 1915, un argument de l'appel à ne pas réparer l'édifice ${ }^{15}$.

Pour comprendre les implications (politiques et patrimoniales) de cette référence récurrente, il faut remonter aux textes qui ont marqué le tournant du siècle précédent (après Chateaubriand, 1811). À commencer par La Prière sur l'Acropole d'Ernest Renan (1883). Les ruines antiques d'Athènes représentent pour lui l'Idéal de la civilisation devant lequel vient s'incliner le Barbare venu du Nord, en qui il se reconnaît avec humilité. Quand Maurice Barrès se rend à son tour sur l'Acropole, en 1900, il récuse la dimension universelle du « miracle grec » avancée par son prédécesseur et lui substitue celle du « miracle français ». Il écrit dans l'épilogue du Voyage de Sparte (1906) :

Je rêve d'un temple dressé par un Phidias de notre race dans un beau lieu français [...] où ma vénération s'accorderait avec la nature et l'art, comme celle des anciens Grecs en présence du Parthénon. (Barrès 1994, 488)

Ce rêve s’incarne de manière paradoxale dans la cathédrale bombardée, qui se charge de toutes les attentes barrésiennes : beauté de l'art exaltant la culture d'une France latine, chrétienne, catholique, mise en péril par le Barbare germanique. Tous les éléments de langage qui serviront à construire le discours anti-allemand sont déjà présents dans cet acte de foi nationaliste d'avant-guerre. Le principe d'équivalence énoncé par Joséphin Péladan en 1917 : « Sus à la langue allemande, pour la vengeance de Reims ! » ${ }^{16}$, en dit long sur la place accordée à l'édifice sacré, chargé d'incarner l'identité même de la nation, comme peut le faire la langue. Et pourtant, contrairement à ceux qui glorifient le « Parthénon français », comme symbole de la nation, Péladan voit dans les cathédrales « plus que des monuments nationaux » car elles appartiennent à l'humanité (Péladan 1917, 255). Elles sont, selon lui, « notre œuvre, pas notre propriété » (Péladan 1917, 258).

15 Le débat est lancé en 1915 dans la revue L’Art et les artistes (« Les Vandales en France », 1 ère série, $n^{\circ} 4,8-15$ ) puis repris en mars 1918 («La guerre par Steinlen », $4^{e}$ série, $n^{\circ} 1,41-47$ ) : publication de lettres de soutien au projet de conserver Notre-Dame de Reims en l'état, approuvé par des artistes (Auguste Rodin) et des hommes de lettres (Edmond Haraucourt, alors conservateur au musée de Cluny).

16 Joséphin Péladan, op. cit., p. 270. 


\section{Disparité du langage des ruines}

Et c'est là un point sur lequel achoppe le discours : est-ce au nom de la nation ou au nom de l'humanité qu'il faut déplorer la perte de ce précieux patrimoine? Comme le fait remarquer Dario Gamboni au sujet des deux guerres mondiales : " les dimensions nationale et supranationale de la notion de patrimoine étaient amenées à entrer en conflit » (Gamboni 2015, 62). En fait, l'une ou l'autre de ces dimensions sera sollicitée en fonction du destinataire des discours : si l'on veut convaincre les états neutres d'intervenir contre l'Allemagne, on fait du monument le bien de l'humanité ; si l'on veut dresser l'opinion du pays contre l'ennemi, alors on l'érige en bien de la nation. Les voix dissidentes, capables de s'affranchir des visées propagandistes sont rares (Romain Rolland, G. B. Shaw ${ }^{17}$ ). Mais, de ces discours partisans et enflammés inspirés par les ruines les plus prestigieuses émerge un fait nouveau : c'est la dissociation entre patrimoine et territoire, qui ouvre la voie au concept de « patrimoine culturel de l'humanité entière » (Unesco 1954), défini par la Convention de La Haye en 1954. La valeur universelle du bien prime sur la notion de propriété nationale. Nous découvrons aujourd'hui les effets pervers de ces intentions pacificatrices, qui font de ces biens revendiqués comme communs par la culture occidentale une cible de choix ${ }^{18}$.

À ces deux dimensions (nationale et mondiale) s'ajoute la dimension locale, vécue dans une relation de proximité et marquée par l'affectivité, l'émotion du souvenir et du deuil. Les coups portés à la maison familiale, au village rural, favorisent le processus d'identification, bien rendu par Jean Giono dans Le Grand troupeau (1931), où les paysans du Midi s'affligent de voir les fermes dévastées du Nord. Les ruines ordinaires atteignent au sacré pour le poète anglais Edmund Blunden, qui évoque avec émotion « cet impérieux sentiment de vénération que les ruines de village éveillaient en [lui] plus vivement peut-être qu'un chef-d'œuvre de Wren ne pourrait le faire aujourd'hui $»^{19}$. À l'inverse, le critique dramatique anglais Edmund Gosse, qui se scandalise de la profanation des monuments français, se félicite que le département des Ardennes, particulièrement éprouvé par

17 Romain Rolland considère Louvain comme « le patrimoine du genre humain » sans mettre les Allemands au ban de l'humanité (Rolland 2013, 49) ; G. B. Shaw accuse la guerre et non l'Allemagne de détruire les cathédrales (voir Pharand 2000, 211-212).

18 On songe à la destruction du temple de Baalshamîn, sur le site archéologique syrien de Palmyre, revendiquée par l'organisation «État islamique » en août 2015. Ce qui est visé est moins la matérialité de ce bien que l'investissement dont il fait l'objet par la culture ennemie, comme l'explique Paul Veyne (2015, 118-119).

19 «[...] that compelled spirit of reverence which those village ruins awoke in me, more vividly perhaps than a Wren masterpiece can today » (Blunden 2010, 54). 
l'invasion, soit l'un des plus pauvres de France en matière d'architecture artistique et historique ${ }^{20}$. Il constate aussi que les dévastations matérielles commises en France par les Allemands (à l'exception de Reims) n'ont pas éveillé la même sympathie en Angleterre que celles perpétrées en Belgique (Gosse 1916, 74).

Ces exemples nous éclairent sur la disparité du langage des ruines à l'épreuve de la guerre : les critères d'appréciation varient en fonction de la situation du locuteur, de son idéologie, des étapes du conflit. Joséphin Péladan qui, avant Maurice Barrès, avait pris la défense des églises rurales menacées par les conséquences de la loi de Séparation, revient sur cet attachement profond au clocher du village : «Sans motif de piété, attiré par le seul effet, le poilu a considéré l'église démolie comme la figure la plus pitoyable de la dévastation » (Péladan 1917, 227-228). La vue des ruines entretient une souffrance partagée qui répare le clivage d'une France divisée par la Loi.

Ainsi, en se reformulant, le langage des ruines entérine les mutations (souvent conflictuelles) qui s'opèrent dans la conscience patrimoniale au cours de ces années de guerre et montre le chemin parcouru depuis la défense du monument historique au XIX ${ }^{\text {e }}$ siècle. Les écrivains qui se sont toujours impliqués pour cette cause participent de cette évolution en cours. C’est le cas de Marcel Proust qui, en bon disciple de John Ruskin, s’alarme « surtout de voir anéantis une telle quantité d'ensembles vivants qui rendaient le moindre village de France instructif et charmant » (Proust 1989, 373). Un changement s'opère dans le mode de relation au cadre architectural, qui passe de l'institutionnel à l'implication individuelle, au sentiment d'appropriation dû à l'investissement affectif provoqué par la perte. L'attachement à la mémoire du lieu, indépendamment de sa valeur d'art ou d'histoire, se manifeste avec une force nouvelle et dans une formulation plus explicite.

\section{Une poétique de la ruine de guerre?}

Le « langage des ruines », dans la citation qui nous a permis d'introduire notre sujet, est donné pour un " langage que les âmes poétiques peuvent seules comprendre ». Il appartiendrait au domaine réservé de la contemplation poétique, déconnecté du présent et coupé de l'histoire ${ }^{21}$. Cette approche romantique paraît

20 « From the purely aesthetic point of view we may congratulate ourselves that Ardennes is one of the poorest departments of France in historical and artistic monuments » (Gosse 1916, 103).

21 Par rapport à la vision progressiste de Volney, qui voyait dans les Pyramides le monument de l'oppression des peuples (voir Mortier 1974, 189), l'approche romantique sollicite l'imagina- 
peu applicable au cas qui nous occupe, le changement de nature de l'objet rendant le langage y afférent caduc et inopérant. Les historiens, d'ailleurs, préfèrent évacuer «la question de la sublimation dans les représentations de ruines » (Danchin 2015, 25) pour se focaliser sur leur réception effective et leur interprétation. Le rappel de la posture romantique, qui apparaît décalée et inappropriée dans le contexte qui nous occupe, nous conduit néanmoins à interroger la place de l'esthétique dans notre analyse. Est-il légitime de parler d'une poétique de la ruine de guerre?

Quand Théophile Gautier décrit Paris, en juin 1871, après les dévastations de la guerre civile, il se surprend à éprouver « une admiration involontaire » : « Par un sentiment que nous reprochera, mais que nous pardonnera tout artiste, parce qu'il l'eût à coup sûr éprouvé, nous fûmes avant tout frappés de la beauté de ces ruines » (Gautier 1881, 327). Les mots « pittoresque, grandiose » reviennent sous sa plume. Même constat chez Jules Claretie : «Épouvante, est-ce bien le sentiment qu'on éprouve ? Non ; le sentiment artistique est si puissant, le désastre a fait de ces choses somptueuses des choses si belles, qu'on s'arrête et qu'on admire » (Claretie 1871, 54-55).

Cette perception esthétisante ne semble plus de mise en 14-18. Du moins n'est-elle plus ouvertement assumée dans les discours. En témoigne le malaise provoqué par la déclaration du poète italien Gabriele D’Annunzio, lors de sa visite à Reims, en avril 1915. Au journaliste du Figaro qui lui demande ses impressions à la vue de la cathédrale gravement endommagée, le poète répond : « La cathédrale n’a jamais été aussi belle. La cathédrale s’achève ». Il répète au cardinal-archevêque de Reims : «La cathédrale s'achève dans les flammes. On a envie de tomber à genoux devant ce miracle [...] Qu'on ne touche pas aux statues, qu'on ne fasse pas de réparations... ». Le narrateur de cette visite mémorable parle de " phrase ahurissante » et de la stupeur du cardinal qui en écarquille les yeux (Chatelle 1951, 134-135). L’anecdote a le mérite de montrer à quel point la perspective adoptée par D’Annunzio était en décalage avec le contexte et aurait pu être irrecevable si l'esthète décadent n'avait pas été aussi un fervent partisan de l'entrée en guerre de l'Italie au côté des Alliés.

Le dramaturge belge Maurice Maeterlinck rejette, en revanche, toute concession au registre du beau, au nom de ses compatriotes : " On ignore l'art de la mise en valeur du sublime. On ne se soucie guère de tirer parti des beautés de la situation » (Maeterlinck, [1936]). La souffrance des victimes est trop récente et trop liée au spectacle des ruines pour ne pas inhiber le sentiment esthétique et le

tion, le rêve, la beauté des formes plutôt que des considérations politiques ancrées dans une conscience historique. 
faire apparaître comme indécent. Le passage de l'esthétisation au pathos libère le langage de l'émotion, plus apte à produire un effet sur la sensibilité du lecteur qu'à mettre en valeur la beauté des formes. La propagande exploite cette ressource, en faisant abondamment circuler des images de ruines (photographies, cartes postales, presse illustrée) et en exposant à Paris des œuvres d'art endommagées ou évacuées de la zone des armées et qui sont censées renvoyer, par métonymie, aux sites dévastés ${ }^{22}$. Mais, sous le couvert de la réprobation morale, de la condamnation du vandalisme allemand, perce la fascination pour ces œuvres « mutilées » données en spectacle. Le poète, romancier et critique Camille Mauclair rédige le compte rendu de cette fameuse exposition du Petit Palais. S’il tient à souligner le scandale de "L'art assassiné ", se conformant au slogan officiel, il n'est pas insensible à la mélancolie ambiante et laisse échapper ces mots : « Et seulement peu à peu, une Beauté éparse naît, se révèle et plane sur cette désolation » (Mauclair 1917, 11). C'est bien la destruction qui fait naître le beau de ces œuvres qui, auparavant, auraient été jugées (selon l'auteur) « artistement banales » (Mauclair 1917, 13). Ce double discours montre que, en dépit des circonstances tragiques, le plaisir esthétique, bien qu'éprouvé comme tabou, fait retour par le pathétique.

On pourrait penser que les écrivains-combattants, qui ne sont pas en situation de spectateurs mais d'acteurs, en tant que témoins in situ de ces dévastations, se sentiraient étrangers à une approche esthétique de la ruine. Or, nombre de descriptions trahissent une séduction implicite et leurs auteurs utilisent pour la communiquer au lecteur le moule de leur tradition littéraire nationale. Edmund Blunden rapporte une scène de contemplation digne de figurer dans la poésie des tombeaux propre à l'Angleterre : dans un cimetière de village bombardé, des soldats fascinés méditent devant les caveaux ouverts par les obus (Blunden 2010, 37). La menace d'une mort prochaine prête au sentiment du sublime qui les étreint une authentique dimension existentielle. L'intensité de ce vécu partagé par l'auteur, vient rehausser la beauté descriptive du passage. Dans les textes français, il arrive qu'une atmosphère de rêverie romantique transfigure la vision des ruines, comme chez Maurice Genevoix : «Par-delà les prés vaporeux, les Éparges, offrant à la clarté nocturne le flanc de toutes leurs ruines, semblent un village de songe qu'un prodige vient de susciter »(Genevoix 2013, 561). Ces exemples montrent que, quelles que soient les circonstances, nul n'échappe à l'emprise de son héritage culturel et que la ligne de démarcation entre les ruines du temps et

22 « Leurs plaies permettent d'imaginer le décor de désolation d'où elles proviennent ». "Ils évoquent les édifices détruits, les cités presque anéanties, les villages incendiés » (Exposition 1916, avant-propos non paginé). Voir également le catalogue de l'exposition 1914 - 1918. Le patrimoine s'en va-t-en guerre (Patrimoine 2016). 
les ruines de guerre est plus poreuse qu'on ne le croit, car l'imaginaire n'est pas cloisonné.

Il reste à savoir dans quel sens la Grande Guerre a fait évoluer la poétique de la ruine. Dans les descriptions les plus novatrices, à la perception statique d'un artefact aux contours stabilisés (la belle ruine), se substitue le processus dynamique de la ruine en train de se faire, c'est-à-dire le spectacle de la destruction en marche. Camille Mauclair tente de rendre compte de cet inexorable mouvement en écrivant : " La ruine s'est propagée, comme un feu, comme une pestilence » (Mauclair 1917, 30). Il s'agira donc, pour l'écrivain témoin, de saisir l'instant de l'écroulement, de rendre palpable la tension entre un avant et un après pour dire le déchaînement de la violence. Ainsi, le romancier américain John Dos Passos, engagé volontaire, assiste au bombardement d'une ancienne abbaye gothique dans la forêt de l’Argonne, détruite en une nuit alors qu'elle avait été préservée pendant des siècles (voir Dos Passos 2003, chap. IV, 25-30 ; trad. fr. Dos Passos 2007, 98-104). Le récit linéaire progresse en trois temps : découverte de l'édifice par une nuit de brume, puis rêve gothique inspiré par le séjour sur les lieux, enfin ravages causés par les tirs d'artillerie. Vient ensuite l'évocation rétrospective des faits, condensés en deux images d'une égale beauté. Lors d'une permission à Paris, le protagoniste du récit s'endort sur le parvis de la cathédrale Notre-Dame et quand, au réveil, la façade et les tours lui apparaissent noyées dans la brume du petit matin, aussitôt surgit le souvenir de l'abbaye :

So had the abbey in the forest gleamed tall in the misty moonlight; like mist, only drab and dense, the dust had risen above the tall apse as the shells tore it to pieces. (Dos Passos 2003, chap. V, 39)

[C'était ainsi, en hauteur, que l'abbaye dans la forêt rayonnait au clair de lune vaporeux ; et, comme un autre brouillard, mais cette fois dense et fauve, la poussière avait enveloppé la haute abside au moment où les obus la mettaient en pièces.] (Dos Passos 2007, 119)

Ramenée à la conscience du témoin par l'analogie, la scène de la destruction est répétée à la faveur de l'analepse. Elle tient sa puissance évocatoire de la brutale succession des faits - grandeur et majesté puis dislocation soudaine de l'architecture - soulignés par la transformation du brouillard en poussière, de la vaporeuse clarté en nuage " dense et fauve ». Ce qui résulte de l'acte destructeur, c'est-àdire la ruine précisément, est absent du texte. Seul l'instant de la pulvérisation importe car il dit l'acmé de la violence guerrière.

L'accélération du temps est perçue non seulement dans l'immédiateté de l'anéantissement, mais également dans le processus d'effacement qui lui succède. Sur le front belge, on peine à reconnaître d'« anciens villages dont les ruines, en 
trois ans, [se sont] couvertes de ronces et d'herbes folles » (La Belgique en guerre [1936]). Les auteurs tentent de rendre le vertige du raccourci temporel alors que la ruine est censée s'inscrire dans la longue durée de l'érosion. Le romancier Gabriel Chevallier s'affole de voir le présent reculer dans le temps, se muer en passé à un rythme accéléré : les « anciens abris [de 1915] ont le mystère poignant des ruines [en 1918] » (Chevallier 2008, 308). Le temps du vécu se transforme en temps archéologique. L'effet « palimpseste » de la ruine de ruine superpose les strates temporelles. Ainsi, la ruine médiévale du donjon de Coucy, longtemps conservée et entretenue, est remplacée par la ruine de guerre, brutale et profanatrice. Le motif est comparable, toutes proportions gardées, à celui des morts tués une seconde fois.

Au fil des années, à mesure que s'affirme la prise de conscience de la radicale nouveauté du conflit : une guerre de matériel, industrielle, mondiale, totale, l'écriture tâtonne à la recherche de formes d'expression inédites pour en rendre compte. S’agissant du rendu de la destruction architecturale, le défi consiste à trouver une forme pour dire l'informe. Car, s'interrogent les auteurs, comment représenter le vide, le rien ? Le désarroi saisit le narrateur d'Henri Barbusse : « Le village a disparu. Jamais je n'ai vu une pareille disparition de village [...] plus rien n’a de forme : il n'y a pas même un pan de mur, de grille, de portail, qui soit dressé [...] » (Barbusse 1972, 194). Pour Pierre Loti, en mission dans la zone des armées, « il n’y a pas de mots » pour transcrire ce qu'il voit car, résume-t-il : « Rien n’a plus forme de rien, nulle part » (Loti 1917, 29). Le réalisme descriptif, impuissant à intégrer la disparition de tout repère formel, est parfois traversé par des fulgurances qui font vaciller le principe de réalité. Élie Faure rapporte ainsi, sur le mode fantastique, sa vision déformée de soldat, la nuit, du fond de la tranchée :

Vu d'en bas les ruines montent, pèsent sur vous, s'emparent de l'espace noir. Encore un village terrible. Les spectres des murs me suivent, des deux côtés du boyau. (Faure 2005, 274)

Dans les romans de la Grande Guerre, la description des ruines n'occupe cependant pas la même place que dans la Trümmerliteratur qui se développera après la Seconde guerre mondiale, notamment en Allemagne, où les paysages de villes dévastées constituent la matière même du récit ${ }^{23}$. La prose narrative issue du

23 Pour illustrer cette caractéristique de la « littérature des ruines », citons, par exemple, la description de Cologne dans Heinrich Böll, Der Engel schwieg, inédit jusqu’en 1992 (Böll 1992 ; trad. fr. Le Silence de l'ange, Böll 1995). 
conflit de 14-18 la plus réceptive aux esthétiques d'avant-garde intègre au récit des fragments descriptifs, selon la technique du collage. La violence de l'expressionnisme porte à son paroxysme la métaphore corporelle de la dislocation architecturale ou encore le découpage cubiste de l'espace montre la distorsion de la perspective vécue par le sujet, placé au cœur de l'action. Quant à la poésie, elle est habitée par des images fortes comme les calvaires et les autels brisés de Wilfred Owen, montrant le corps christique mutilé (« Le christianisme », Owen 2012, 32-33 ; « At a Calvary near the Ancre », Owen 2012, 38-39).

\section{Conclusion}

La poétique des ruines qui s'élabore dans les écrits de la Grande Guerre, qu'elle émane des témoins de l'arrière ou des combattants du front, donne tout son sens à ce que Chateaubriand présentait comme une " poétique des morts » (Chateaubriand 1966, 40). Non pas celle qu'inspire l' " ouvrage du temps » (ibid.), associé aux notions de mélancolie, de charme, de plaisir esthétique lié à la présence vivante de la nature, mais celle qui résulte de l' " ouvrage des hommes » (ibid.) et qui ne parle que de "dévastations " et de " malheur » (ibid.). C'est bien à cette source que puisent discours de protestation et récits de guerre pour tenter de rendre compte de la violence faite aux choses : «l'image du néant, sans une puissance réparatrice » (ibid.), comme le résumait sans concession l'auteur romantique. De telles ruines tiennent leur éloquence de la tragédie vécue par les hommes. Elles sont le signe tangible de la destinée des corps anonymes, dérobés au regard et soustraits à la mémoire. Villages disparus, bâtisses disloquées, architectures mutilées racontent la souffrance endurée, amplifiée par la perte et le deuil. La démesure et la désolation des paysages de ruines, soulignées dans tous les écrits, donnent une traduction concrète et durable au phénomène nouveau de la mort de masse. L’intégration des données inédites de la Première guerre mondiale contribue ainsi à la réécriture du « langage des ruines », qui perpétue la trace du traumatisme collectif.

\section{Bibliographie}

L'Art et les artistes. "Les Vandales en France », 1 ère série, no 4 (1915). « L'Art assassiné », $2^{\mathrm{e}}$ série, $n^{\circ} 5$, (février 1917). "La guerre par Steinlen », $4^{e}$ série, no 1 (mars 1918).

Barbusse, Henri. Le Feu, Journal d'une escouade. Paris : E. Flammarion, 1916. Paris : J'ai Lu, 1972. 
Barrès, Maurice. Voyage de Sparte [1906]. Romans et voyages. Paris : Robert Laffont, 1994. 379-508.

Barrès, Maurice. «La leçon fortifiante des ruines ». La Lorraine dévastée (26 sept 1914). Paris, Félix Alcan, 1919. 1-6.

La Belgique en guerre. Album illustré. Textes de Maurice Maeterlinck et Louis Dumont-Wilden. Nouvelle édition publiée par Ernest Van Hammée. Bruxelles : s. d. [1936], non paginé.

Blunden, Edmund. Undertones of War. Londres : Penguin Classics, 2010 [1928].

Böll Heinrich. Der Engel schwieg. Cologne : Kiepenheuer \& Witsch, 1992 [1949/1950, inédit]. Böll, Heinrich. Le Silence de l'ange. Alain Huriot (trad.). Paris : Le Seuil, 1995.

Chateaubriand, François-René de. Génie du christianisme [1802]. Troisième partie, Livre cinquième, chapitre III, « Des ruines en général ». Paris : Garnier-Flammarion, 1966. 40-42.

Chatelle, Albert. Reims, ville des sacres, notes diplomatiques secrètes et récits inédits, 1914 1918. Paris : impr. de Téqui, 1951.

Chevallier, Gabriel. La Peur. [Mayenne], Le Dilettante, 2008 [1930].

Claretie, Jules. «L'Hôtel de Ville ». L'lllustration (22 juillet 1871) : 54-55.

Danchin, Emmanuelle. Le Temps des ruines 1914-1921. Rennes : Presses Universitaires de Rennes, 2015.

Deuxième conférence internationale de la paix, 1907. Paris : Imprimerie nationale, 1908.

[Consultable sur Gallica : https://gallica.bnf.fr/ark:/12148/bpt6k56139030/f1.item.r=209]

Dorgelès, Roland. Le Réveil des morts. Cuise-la-Motte : Le Trotteur ailé, 2010 [1923].

Dos Passos, John. One Man's Initiation: 1917 [1920]. Novels 1920-1925. New York : The Library of America, 2003. 3-85.

Dos Passos, John. L'Initiation d'un homme : 1917. Marc Freeman (trad.). Paris : Éditions Rieder, 1925. [Rééd. Paris : Gallimard, coll. Folio, 2007].

[Exposition 1916] Exposition d'œuvres d'art mutilées ou provenant des régions dévastées par l'ennemi. Paris : D. Niestlé, 1916.

Faure, Élie. La Sainte-Face. Carine Trévisan (éd.). Paris : Bartillat, 2005.

Gamboni, Dario. La destruction de l'art : iconoclasme et vandalisme depuis la Révolution française. Estelle Beauseigneur (trad.). Dijon : Les Presses du réel, 2015.

Gautier, Théophile. Tableaux de siège. Paris, 1870-1871. Paris : Charpentier, 1881.

Genevoix, Maurice. La Boue. In Ceux de 14. Paris : Flammarion, 2013. 433-637.

Gosse, Edmund. «The Desecration of French Monuments ». Inter Arma. Being Essays Written in time of War. London : Heineman, 1916. 73-105.

Harlaut, Yann. «L'incendie de la cathédrale de Reims, 19 septembre 1914 : Fait imagé ... Fait imaginé ». Mythes et réalités de la cathédrale de Reims de 1825 à 1975. Paris : Somogy, 2001. 71-79.

Kipling, Rudyard. France at War. Londres : Macmillan and Co, 1915.

Kipling, Rudyard. La France en guerre. Laurent Bury (trad.). Paris : Les Belles Lettres, 2014.

La Sizeranne, Robert de. «Les ruines ». L'Illustration (18 décembre 1915) : non paginé.

La Sizeranne, Robert de. «Ce que la guerre enseigne aux peintres - À propos du Salon de 1918 ». Revue des deux mondes, t. 45 (1918) : 610-634.

Lavedan, Henri. Jeunes ruines. Préface de Selthic 1917, non paginée.

Lavedan, Henri. Les Grandes heures. Première série (août 1914-janvier 1915). Paris : Perrin, 1919.

Lee, Vernon. "In Time of War (Summer, 1917)». The Golden Keys and Other Essays on the Genius Loci. Londres : John Lane, The Bodley Head, 1925. 241-249. 
Loti, Pierre. L'Outrage des barbares. Paris : impr. de G. de Malherbe \& Cie, 1917.

Maeterlinck, Maurice. Les Débris de la guerre. Paris : Charpentier, 1916.

Maeterlinck, Maurice. «La résistance du peuple belge ». La Belgique en guerre. Bruxelles :

Ernest Van Hammée, s. d. [1936]. [Album non paginé].

Mâle, Émile. "La cathédrale de Reims " [octobre 1914]. L'Art allemand et l'Art français du Moyen Âge. Paris : Armand Colin, 1918. 220-222.

Mauclair, Camille. "L'art assassiné ». L’Art et les artistes, "L’Art assassiné », 2e série, n5, (février 1917). 7-33.

Michaud, Joseph-François, et Jean-Joseph-François Poujoulat. Correspondances d'Orient, 1830-1831. Vol. 4. Bruxelles : Gregoir, Wouters et Cie, 1841.

Mortier, Roland. La Poétique des ruines. Genève : Droz, 1974.

Owen, Wilfred. Et chaque lent crépuscule ... Xavier Hanotte (éd. et trad.). Bègles : Le Castor astral, 2012.

[Patrimoine 2016] 1914-1918. Le patrimoine s'en va-t-en guerre. Cat. exp. Jean-Marc Hofman (dir.). Paris : Cité de l'Architecture et du Patrimoine, Norma Éditions, 2016.

Péladan, Joséphin. L’Art et la guerre. Paris : E. de Boccard, 1917.

Pharand, Michel W. Bernard Shaw and the French. Gainesville : University Press of Florida, 2000.

Proust, Marcel. À la Recherche du temps perdu. Vol. IV Le Temps retrouvé [1927]. Jean-Yves Tadié (éd.). Paris : Gallimard, Bibliothèque de la Pléiade, 1989.

Riegl, Alois. Der moderne Denkmalkultus. Vienne et Leipzig : W. Braumüller, 1903.

Riegl, Aloïs. Le Culte moderne des monuments. Son essence et sa genèse. Daniel Wieczorek (trad.). Paris : Seuil, 1984.

Rolland, Romain. Au-dessus de la mêlée. Paris : Paul Ollendorff, 1915. Paris : Payot, 2013.

Rostand, Edmond. « La Cathédrale ». Le Figaro (9 octobre 1914). In Les Poètes de la Guerre, Paris : Berger-Levrault, 1915. 115.

Selthic, André. Jeunes Ruines. Poëmes de guerre. Illustrés par Mathurin Meheut. Paris : Jules Meynial, 1917.

[Unesco 1954] Convention pour la protection des biens culturels en cas de conflit armé, avec Règlement d'exécution 1954.

http://portal.unesco.org/fr/ev.php-URL_ID=13637\&URL_DO=DO_TOPIC\&URL_SECTION=201. html (8 juin 2016).

Valéry, Paul. «La Crise de l'esprit » [1919]. Variété. CEuvres I. Paris : Gallimard, Bibliothèque de la Pléiade, 1957. 988-1000.

Veyne, Paul. Palmyre. L'irremplaçable trésor. Paris : Albin Michel, 2015.

Zola, Émile. La Débâcle [1892]. Paris : Gallimard, coll. Folio Classique, 1984.

Zola, Émile. Lourdes [1894]. Paris : Gallimard, coll. Folio Classique, 1995.

Zola, Émile. Rome [1896]. Paris : Gallimard, coll. Folio Classique, 1999.

Joëlle Prungnaud holds a Doctor degree in Comparative Literature from Paris IVSorbonne University. Since her Ph.D. thesis Gothique et Décadence : Recherches sur la continuité d'un mythe et d'un genre au XIX $X^{e}$ siècle en Grande-Bretagne et en 
France (Paris: Honoré Champion, 1997, 2015), she has refocused her research on the relation between literature and architecture: Figures littéraires de la cathédrale (1880 - 1918) (Lille: Presses Universitaires du Septentrion, 2008). She taught Comparative Literature at the University of Lille from 1994 to 2012. Currently a Professor Emerita, she is interested in the writers' view of destructions during WWI, both in the French- and English-speaking world: "Writers' Response to the Architectural Destructions of the Great War”. Journal of War \& Culture Studies 9.3 (2016). London: Routledge. 237-251. 
